\title{
Multi-modal Ultrasonic Diagnosis and Prognosis for Primary Thyroid Lymphoma Invasiveness
}

\section{Chenjuan Peng}

1.The Cancer Hospital of the University of Chinese Academy of Sciences (Zhejiang Cancer Hospital) 2.Institute of Basic Medicine and Cancer (IBMC),Chinese Academy Of Sciences

\section{Chen Yang}

1.The Cancer Hospital of the University of Chinese Academy of Sciences (Zhejiang Cancer Hospital) 2.Institute of Basic Medicine and Cancer (IBMC), Chinese Academy Of Sciences

\section{Jincao Yao}

1.The Cancer Hospital of the University of Chinese Academy of Sciences (Zhejiang Cancer Hospital) 2.Institute of Basic Medicine and Cancer (IBMC), Chinese Academy Of Sciences

\section{LingYan Zhou}

1.The Cancer Hospital of the University of Chinese Academy of Sciences (Zhejiang Cancer Hospital) 2.Institute of Basic Medicine and Cancer (IBMC), Chinese Academy Of Sciences

\section{Jingjing Xu}

1.The Cancer Hospital of the University of Chinese Academy of Sciences (Zhejiang Cancer Hospital) 2.Institute of Basic Medicine and Cancer (IBMC),Chinese Academy Of Sciences

\section{Junzhou Wu}

1.The Cancer Hospital of the University of Chinese Academy of Sciences (Zhejiang Cancer Hospital) 2.Institute of Basic Medicine and Cancer (IBMC),Chinese Academy Of Sciences

\section{Jiazheng Zhao}

1.The Cancer Hospital of the University of Chinese Academy of Sciences (Zhejiang Cancer Hospital) 2.Institute of Basic Medicine and Cancer (IBMC), Chinese Academy Of Sciences

\section{Shuailing Peng}

1.The Cancer Hospital of the University of Chinese Academy of Sciences (Zhejiang Cancer Hospital) 2.Institute of Basic Medicine and Cancer (IBMC), Chinese Academy Of Sciences

\section{Dong Xu ( $\nabla$ xudong@zjcc.org.cn )}

1.The Cancer Hospital of the University of Chinese Academy of Sciences (Zhejiang Cancer Hospital) 2.Institute of Basic Medicine and Cancer (IBMC), Chinese Academy Of Sciences

\section{Research Article}

Keywords: primary thyroid lymphoma, multi-modal, ultrasonic pattern, invasiveness, prognosis

Posted Date: June 30th, 2021 
DOI: https://doi.org/10.21203/rs.3.rs-629680/v1

License: (c) (1) This work is licensed under a Creative Commons Attribution 4.0 International License. Read Full License 


\section{Abstract}

Objective Sixty-nine patients with primary thyroid lymphoma $(P T L)$ were evaluated for diagnostic value and prognosis for PTL invasiveness.

Methods We retrospectively (2008-2019) analyzed multi-modal ultrasonic images and clinical characteristics from pathologically confirmed PTL patients. These patients were divided into aggressive PTL $(n=46)$ and indolent PTL $(n=23)$.

Results Age( $>70$ years old) and elevated LDH (lactase dehydrogenase) were statistically different clinical features between aggressive and indolent PTL. From ultrasonic images, 34 cases were nodular, 11 diffused, and 24 mixed. Mixed types displayed high invasiveness (45.7\%) while diffuse types displayed higher inertness (39.1\%), and differences were statistically significant $(P=0.000)$. Elastography, invaded thyroid capsule and increased chaotic vascularity also showed significant differences between aggressive and indolent PTL. We observed statistical difference in OS(overall survival rates) between aggressive and indolent PTL $(p=0.032)$. Single factor Kaplan-Meier $(K-M)$ analysis showed that: age $>70$ years old, aggressive pathology, Ki67 $>30 \%$, elastography scored $>3$ were positively correlated with the risk of poor prognosis of PTL $(\mathrm{P}<0.05)$.

Conclusions Multi-modal ultrasound provides accurate ultrasonographic information, e.g., ultrasound patterns, elastography, invaded thyroid capsules, and hypervascularity, which facilitates PTL invasiveness diagnostics for improved clinical treatment. In addition, PTL patients with age $>70$ years old, aggressive pathology, Ki67>30\%, elastography scored $>3$ are more likely to have poor prognosis.

\section{Introduction}

Primary thyroid lymphoma (PTL) is rarely observed, accounting for $2-5 \%$ of primary thyroid malignancies[1, 2]and $2 \%$ of all extranodal lymphomas[3]. PTL is usually B-cell-derived Non-Hodgkin lymphoma, the most common type being diffuse large B cell lymphoma (DLBCL) and followed by mucosa-associated lymphoid tissue lymphoma (MALT). Currently, a PTL diagnosis mainly depends on pathology, however, its preoperative diagnosis accuracy is not high. PTL is often misdiagnosed as thyroid carcinoma, Hashimoto's thyroiditis (HT), nodular goiter, and other diseases. Multi-modality ultrasonography including elastosonography, blood flow signal, and contrast-enhanced ultrasonography (CEUS) are useful non-invasive techniques widely used for liver, breast, and thyroid diagnostics[4-6].

Multi-modal ultrasonic and clinical features from 69 patients were pathologically confirmed as PTL, and retrospectively analyzed in this study. We sought to improve the diagnostic accuracy of PTL, explore the diagnostic value of ultrasound classification for aggressive primary lymphomas, and perform prognostic analysis.

\section{Materials And Methods}




\section{Patients}

From 2008 to 2019, 69 patients, pathologically confirmed with PTL, were retrospectively included in this study. Patients included 27 males and 42 females, with ages ranging from 38-83 years and an average age of $62.2 \pm 11$ years. Examinations showed that only the neck and upper mediastinal lymph nodes or no lymph nodes were involved. Medical records were also checked, and laboratory findings (lactate dehydrogenase(LDH), erythrocyte sedimentation rate(ESR), free T3, free T4, TSH and so on), and pathological results were recorded. Tissues were diagnosed using pathological and immunohistochemical analyses. All patients were pathologically classified according to the 2016 World Health Organization classification standard[7].

\section{Classification of PTL}

Aggressive PTL includes DLBCL (diffuse large B cell lymphoma) ,FLIII (follicular lymphoma III) 口Burkitt (Burkitt lymphoma), DLBCL with MALT. Indolent PTL contains MALT (Mucosa-associated marginal zone B-cell lymphoma) only.

\section{Instrumentation}

A Color Doppler Ultrasonic Diagnosis Apparatus was used in this study (GE Logiq E9, U.S.; Philips IU22, Netherlands; Toshiba type, Japan, etc.), with a probe frequency of $5.0 \mathrm{MHz}-14.0 \mathrm{MHz}$, which were all linear array probes. Multi-section ultrasound scans were conducted at the thyroid gland at the front of the neck. The probe frequency, depth, gain, and color blood flow scales were selected according to specific situations. All data were stored as digital images for further analysis.

\section{Imaging Analysis}

Sonographic features including thyroid size, thyroid background echotexture, lesion size, boundary, aspect ratio, elastosonography[8], calcification, vascularity, invaded thyroid capsules, cervical lymphadenopathy of Each type were retrospectively determined by two radiologists (CJ.P and C.Y) in consensus manner. Each of the radiologists had over 1500 cases thyroid sonography experience. If multifocal nodular was present in patients, the largest mass was selected or analysis. Elastic image was scored (1-5 points) using colors displayed in the ROI. When elastography points were $>3$, hard nodules were indicated[9]. When lymph nodes were roundly enlarged, the hyperechoic structure of the lymphatic hilum disappeared, the internal echo was uneven, and irregular blood flow signals were seen inside, abnormally enlarged lymph nodes were recorded[10].

\section{Contrast-enhanced ultrasound (CEUS)}

The ultrasonic diagnostic device (GE Logiq E9, U.S.) was used for CEUS, with a probe frequency of 7.5 $\mathrm{MHz}-15 \mathrm{MHz}$ and $2.4 \mathrm{ml}$ SonoVue ${ }^{\mathrm{TM}}$ (Bracco, Milan, Italy) microbubbles. After observing lesion morphological features and blood supply using conventional 2D and color Doppler ultrasound, a preliminary diagnosis was made. Then the patient was definitively diagnosed using CEUS according to 
guidelines[11]. During the process, lesion location was continuously and dynamically observed from entering the lesion to completely disappearing in almost 2 min.

\section{Survival analysis}

Follow-up visits were conducted by telephone and were based on medical records. Overall survival (OS) referred to the time of diagnosis to death, or the end of follow-up. Progression-free survival (PFS) referred to the time of diagnosis to the end of progression or follow-up visit. The study deadline was October 31, 2020.

\section{Statistical analysis}

All statistical calculations were completed using SPSS16.0 software. Continuous variables were summarized as means + STD and categorical variables as percentages. Categorical data were compared using chi-square or Fisher's exact tests. The Mann-Whitney test was performed to compare continuous variables. OS/PFS times were calculated by the Kaplan-Meier method, and the Log-rank test was used for comparisons between groups. Multiple factors were tested using Cox regression analysis. Differences were statistically significant at $\mathrm{P}<0.05$.

\section{Results}

\section{Clinical features}

Patient pathological types comprised non-Hodgkin's lymphomas, with 46 aggressive PTL and 23 indolent cases (Table 1). Of these, aggressive lymphomas included five Burkitt lymphoma cases (Figs. 1), four transformation type cases (also called mixed types; DLBCL with MALT), 35 DLBCL cases (Fig. 2), and two follicular lymphoma (FL) grade Illa (FL IIla) cases. Indolent lymphoma comprised 23 MALT cases (Fig. 3). The age of aggressive lymphoma was older in patients, mostly over 70 years old, and was statistically different to indolent PTL. Aggressive lymphoma was usually defined as ki67 $>30 \%[12]$ and was consistent with the pathological type $(P=0.000)$. Of these, the pathological types of five cases were complicated by papillary thyroid carcinoma (PTC), four cases with MALT, and one case with DLBCL. Forty-two cases (61\%) had chronic thyroiditis (Hashimoto's disease). Approximately $26 \%$ of patients with primary lymphoma had hypothyroidism, and $91 \%$ had aggressive PTL. Patients with aggressive lymphoma had higher lactate dehydrogenase (LDH) levels, which were statistically higher than patients with indolent lymphoma. 
Table 1

Comparison of clinical features between aggressive PTL and indolent PTL

\begin{tabular}{|c|c|c|c|}
\hline Index [case(\%)] & $\begin{array}{l}\text { Aggressive PTL } \\
(n=46)\end{array}$ & $\begin{array}{l}\text { Indolent PTL } \\
(n=23)\end{array}$ & $P$ value \\
\hline Gender & $16(34.8)$ & $11(47.8)$ & 0.216 \\
\hline Male & $30(65.2)$ & $12(52.2)$ & $0.040 *$ \\
\hline Female & $64.07 \pm 10.87(45-83)$ & $58.35 \pm 10.37(38-75)$ & $0.02 *$ \\
\hline Aget & $30(65.2)$ & 21(91.3) & $0.041 *$ \\
\hline$\leq 70$ years old & $16(34.8)$ & $2(8.7)$ & $0.039 *$ \\
\hline$>70$ years old & 19(41.3) & $13(56.5)$ & $0.000 *$ \\
\hline Elevated LDH & 18(39.1) & $3(13)$ & $0.012^{*}$ \\
\hline - & $45(97.8)$ & 19(21.3) & 0.468 \\
\hline+ & $1(3.3)$ & $4(1.7)$ & 0.181 \\
\hline Combined with PTC & $4(8.7)$ & 18(78.3) & 1.000 \\
\hline- & $34(73.9)$ & $0(0)$ & 0.929 \\
\hline+ & $36(78.3)$ & $11(47.8)$ & \\
\hline Ki67 & $6(13)$ & $9(39)$ & \\
\hline$\leq 30 \%$ & $8(17.4)$ & $2(8.7)$ & \\
\hline$\varangle 30 \%$ & $26(56.5)$ & $14(60.9)$ & \\
\hline CD3 & $26(56.5)$ & $16(69.6)$ & \\
\hline- & $10(21.7)$ & $1(4.3)$ & \\
\hline+ & $5(10.9)$ & $1(4.3)$ & \\
\hline Hashimoto's thyroiditis & $8(17.4)$ & $1(4.3)$ & \\
\hline- & $22(47.8)$ & $11(47.8)$ & \\
\hline+ & $20(43.5)$ & $11(47.8)$ & \\
\hline Hypothyroidism & $4(8.7)$ & $1(4.3)$ & \\
\hline \multicolumn{4}{|c|}{ Note: Values are presented as mean \pm std(range) or number (\%) } \\
\hline \multicolumn{4}{|c|}{$\begin{array}{l}\text { Unless otherwise indicated, data are numbers of patients, with counted percentage in parentheses. } \\
\text { tData are as mean } \pm \text { standard deviation, with ranges in parentheses. }\end{array}$} \\
\hline \multicolumn{4}{|c|}{ *, $\mathrm{P}<0.05$, difference was statistically significant. } \\
\hline Italics indicate incomple & (<69 cases). & & \\
\hline
\end{tabular}




\begin{tabular}{|c|c|c|c|}
\hline Index [case(\%)] & $\begin{array}{l}\text { Aggressive PTL } \\
(n=46)\end{array}$ & $\begin{array}{l}\text { Indolent PTL } \\
(\mathrm{n}=23)\end{array}$ & $P$ value \\
\hline \multicolumn{4}{|l|}{$\begin{array}{l}- \\
+\end{array}$} \\
\hline \multicolumn{4}{|l|}{ ESR } \\
\hline \multicolumn{4}{|l|}{-} \\
\hline \multicolumn{4}{|l|}{+} \\
\hline \multicolumn{4}{|l|}{ Treatment } \\
\hline \multicolumn{4}{|l|}{ Single treatment } \\
\hline \multicolumn{4}{|l|}{ Combined treatment } \\
\hline \multicolumn{4}{|l|}{ Rejected treatment } \\
\hline \multicolumn{4}{|c|}{ Note: Values are presented as mean \pm std(range) or number (\%) } \\
\hline \multicolumn{4}{|c|}{$\begin{array}{l}\text { Unless otherwise indicated, data are numbers of patients, with counted percentage in parentheses. } \\
\text { tData are as mean } \pm \text { standard deviation, with ranges in parentheses. }\end{array}$} \\
\hline \multicolumn{4}{|c|}{$\star, P<0.05$, difference was statistically significant. } \\
\hline Italics indicate incom & (<69 cases) & & \\
\hline
\end{tabular}

\section{Multi-modal ultrasound features}

Of the 69 pathological cases, 22 underwent ultrasound-guided needle biopsy and 47 cases had pathological tissue from surgical procedures. Ultrasonic characteristics are shown (Table 2). We observed that ultrasonic features of nodular thyroid lymphoma, with different pathological types, included significant internal hypoecho, internal cord-like or grid-like hyperecho, enhanced posterior echo, a large tumor diameter of $>2 \mathrm{~cm}$, a non-imbalanced aspect ratio, less internal calcification, and blood flow signals in the mass. Ultrasonography had a higher positive predictive value for aggressive lymphoma. PTL was divided into three ultrasonic features; diffuse, nodular, and mixed types, based on Ota et al.[13]. Of these, 19 cases were diffuse, 41 nodular, and 14 mixed types. Based on our data, the ultrasound patterns of aggressive and indolent thyroid lymphomas were statistically different $(P<0.05)$. For mixed ultrasound classifications, the proportion of aggressive thyroid lymphomas (45.7\%) was significantly higher than indolent lymphomas $(13 \%, P=0.000)$, while for diffuse ultrasound classifications, the proportion of aggressive thyroid lymphomas $(4.3 \%)$ was significantly reduced when compared with indolent lymphomas $(39.1 \%, P=0.000)$. The thyroid capsule invasion of invasive lymphomas was more likely to be detected $(P=0.007)$. Blood flow signals of nodules in aggressive PTL were higher than for indolent PTL. From nodular ultrasound classifications, most lesions in aggressive lymphomas were hard, 
while indolent lymphomas were softer $(P=0.000)$. Lesions in CEUS were diffuse, non-uniform, mildly enhanced, and some echo-free space was seen locally (Fig. 3). 
Table 2

Comparison of ultrasonic features between aggressive PTL and indolent PTL

\begin{tabular}{|c|c|c|c|}
\hline Index [case(\%)] & $\begin{array}{l}\text { Aggressive PTL } \\
(n=46)\end{array}$ & $\begin{array}{l}\text { Indolent PTL } \\
(\mathrm{n}=23)\end{array}$ & $P$ value \\
\hline Ultrasound pattern & $2(4.3)$ & $9(39.1)$ & $0.000 *$ \\
\hline Diffuse type & $23(50)$ & 11(47.8) & $0.007 *$ \\
\hline Nodular type & $21(45.7)$ & $3(13)$ & $0.007 *$ \\
\hline Mixed type & $1(9)$ & $3(27.3)$ & $0.000 *$ \\
\hline Blood flow signal (Nodular) & $1(0)$ & $4(36.4)$ & 0.057 \\
\hline 0 & 16(69.6) & $3(27.3)$ & 0.430 \\
\hline 1 & $5(21.7)$ & $1(9)$ & 0.218 \\
\hline 2 & $38(82.6)$ & $23(100)$ & 1.000 \\
\hline 3 & $8(17.4)$ & $0(0)$ & 0.226 \\
\hline Invaded thyroid capsule & $9(39)$ & $0(0)$ & 0.226 \\
\hline- & $14(61)$ & 11(100) & 0.324 \\
\hline+ & 15(32.6) & $13(56.5)$ & 1.000 \\
\hline Elastograph & $31(67.4)$ & $10(43.5)$ & 0.559 \\
\hline (Nodular) & 13(28.3) & $6(26.1)$ & 0.160 \\
\hline$>3$ & $21(45.7)$ & $7(30.4)$ & \\
\hline$\leq 3$ & $10(21.7)$ & $9(39.1)$ & \\
\hline Asymmetric enlargement & $2(4.3)$ & $1(4.3)$ & \\
\hline - & $47.04 \pm 15.81(18-80)$ & $40.31 \pm 15.14(20-74)$ & \\
\hline+ & $0(0)$ & $0(0)$ & \\
\hline Tumor position & $23(100)$ & $11(100)$ & \\
\hline Left lobe & 7(30.4) & $6(54.5)$ & \\
\hline \multicolumn{4}{|c|}{ Note: When Elastography points $>3$, it indicates that the nodules are hard. } \\
\hline \multicolumn{4}{|c|}{$\begin{array}{l}\text { Unless otherwise indicated, data are numbers of patients, with counted percentage in parentheses. } \\
\text { tData are as mean } \pm \text { standard deviation, with ranges in parentheses. }\end{array}$} \\
\hline \multicolumn{4}{|c|}{ *, $P<0.05$, difference was statistically significant. } \\
\hline \multicolumn{4}{|c|}{ (nodular) means compared among nodular type PTL. } \\
\hline Italics indicate incomplete $\mathrm{d}$ & & & \\
\hline
\end{tabular}




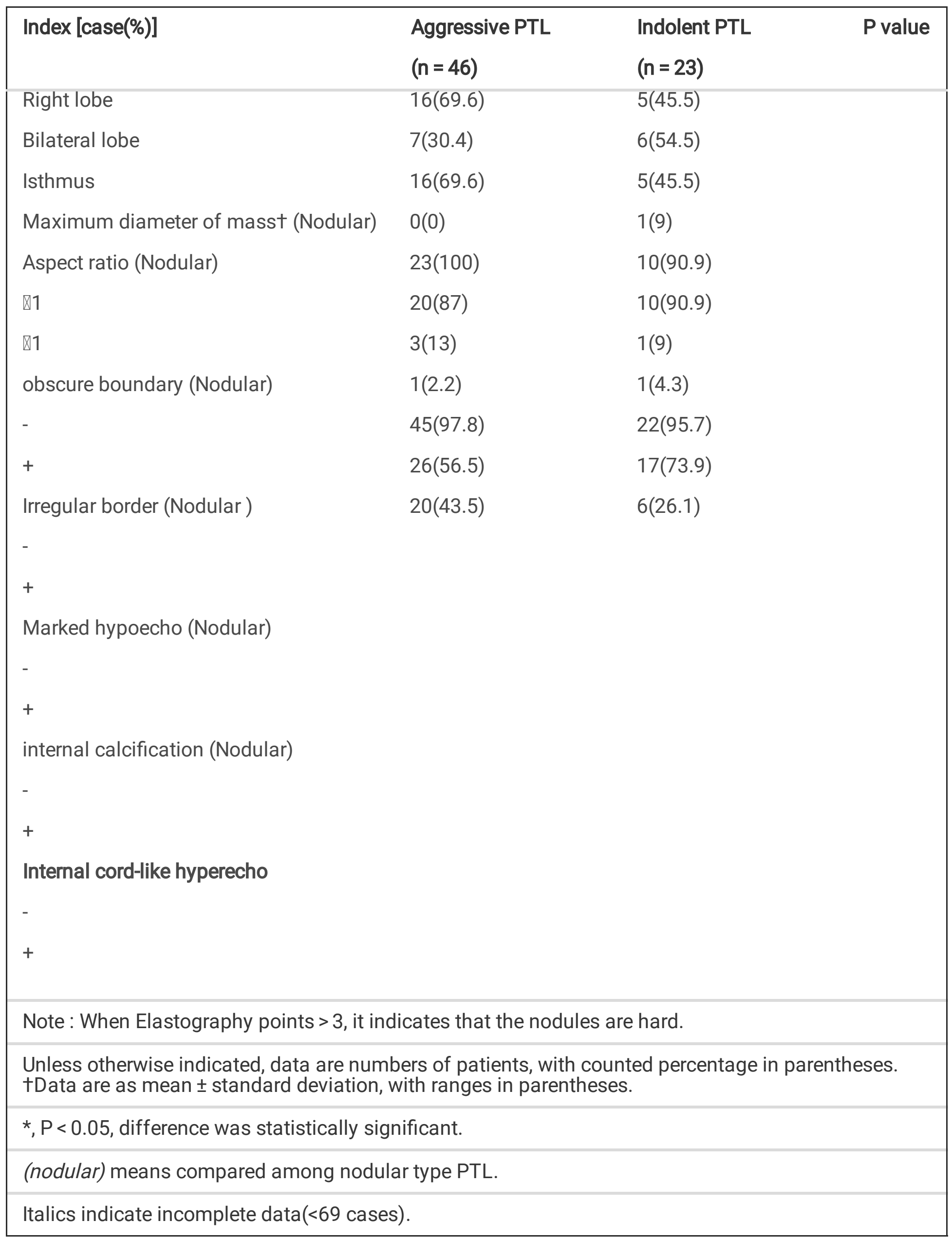




\begin{tabular}{|l|l|}
\hline Index [case(\%)] & \multicolumn{1}{c|}{$\begin{array}{l}\text { Aggressive PTL } \\
(\mathbf{n}=\mathbf{4 6})\end{array}$} \\
\hline Abnormal lymph nodes \\
- \\
\hline Note : When Elastography points $>3$, it indicates that the nodules are hard. \\
\hline $\begin{array}{l}\text { Unless otherwise indicated, data are numbers of patients, with counted percentage in parentheses. } \\
\text { DData are as mean } \pm \text { standard deviation, with ranges in parentheses. }\end{array}$ \\
\hline *, P<0.05, difference was statistically significant. \\
\hline (nodular) means compared among nodular type PTL. \\
\hline Italics indicate incomplete data(<69 cases). \\
\hline
\end{tabular}

\section{Survival analysis}

At follow-up end, the 5-year OS rate for the 69 patients was $92.8 \% \pm 3.1 \%$, and the 5 -year PFS for all 69 patients with PTL was $97.0 \% \pm 2.1 \%$. The 5 -year OS rate for aggressive lymphoma $(n=46)$ and indolent lymphoma $(n=23)$ was $89.1 \% \pm 4.6 \%$ and $100.0 \% \pm 0.0 \%$, respectively $(P=0.032)$ (Fig. $4 A)$. The 5 -year PFS for patients with aggressive and indolent lymphoma was $95.3 \% \pm 3.2 \%$ and $100.0 \% \pm 0.0 \%$, respectively $(P=0.415)$ (Fig. 4B).

Single factor Kaplan-Meier (K-M) analysis showed that: age > 70 years old, aggressive pathology, Ki67 > $30 \%$, elastography scored $>3$ were positively correlated with the risk of poor prognosis of $\mathrm{PTL}(\mathrm{P}<$ 0.05). These results are shown in Table 3. However, there was no evidence to show that the gender, elevated LDH, hashimoto's thyroiditis, diameter, abnormal lymph nodes, invaded thyroid capsule, asymmetric enlargement and blood flow were significantly associated with the prognosis of PTL $(P>$ $0.05)$ using single factor K-M survival analysis. The characteristics $(P<0.05)$ were used to construct a multivariable cox regression equation and the results showed that age (Hazard Ratio(HR) $=15.62,95 \%$ Confidence Interval $(\mathrm{Cl}) 1.79-136.64, \mathrm{P}=0.013$ ) was independent risk factor for PTL. These results are also shown in Table 3. 
Table 3

Prognostic factors for overall survival of PTL.

\begin{tabular}{|c|c|c|c|c|c|}
\hline $\begin{array}{l}\text { Univariate } \\
\text { Factors } \\
\text { (K-M) }\end{array}$ & Cases & $\begin{array}{l}5 \text { year } \\
\text { OS(\%) }\end{array}$ & $\begin{array}{l}\mathrm{P} \\
\text { value }\end{array}$ & $\begin{array}{l}\text { Multivariate } \\
\text { factors } \\
\text { (Cox) }\end{array}$ & $\begin{array}{l}\text { OS Hazard ratio } \\
\text { [95\% Cl] (P value) }\end{array}$ \\
\hline Gender & 27 & $\begin{array}{l}92.6 \pm \\
5.9\end{array}$ & 0.784 & & $15.62[1.79-136.64]$ \\
\hline Male & 42 & & $0.000 *$ & Pathological & $\left(0.013^{*}\right)$ \\
\hline Female & 51 & $4.6^{-4}$ & $0.032^{\star}$ & Ki67 & $\begin{array}{l}0.00[0.00-5.829 E+275] \\
(0.97)\end{array}$ \\
\hline $\begin{array}{l}\text { Age } \\
<70 \text { vears old }\end{array}$ & 18 & $\begin{array}{l}96.1 \pm \\
2.7\end{array}$ & $\begin{array}{l}0.417 \\
0.820\end{array}$ & Elastograph & $\begin{array}{l}45139.91[0.00-1.296 \mathrm{E}+ \\
1321(0.94)\end{array}$ \\
\hline$>70$ years old & 23 & $\begin{array}{l}77.8 \pm \\
9.8\end{array}$ & $0.016^{*}$ & & $\begin{array}{l}157077.52[0.00-1.029 E+ \\
240](0.97)\end{array}$ \\
\hline $\begin{array}{l}\text { Pathological } \\
\text { diagnosis }\end{array}$ & $\begin{array}{l}64 \\
5\end{array}$ & $\begin{array}{l}89.1 \pm \\
4.6\end{array}$ & 0.491 & & \\
\hline $\begin{array}{l}\text { aggressive } \\
\text { indolent }\end{array}$ & 32 & $\begin{array}{l}100 \pm \\
0.0\end{array}$ & 0.139 & & \\
\hline Combined with PTC & 21 & $\begin{array}{l}92.2 \pm \\
3.4\end{array}$ & 0.153 & & \\
\hline 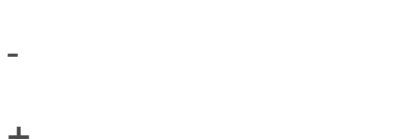 & 34 & $\begin{array}{l}100 \pm \\
0.0\end{array}$ & 0.886 & & \\
\hline Elevated LDH & 10 & $\begin{array}{l}87.5 \pm \\
5.8\end{array}$ & 0.215 & & \\
\hline - & $\begin{array}{l}40 \\
42\end{array}$ & $\begin{array}{l}90.2 \pm \\
6.6\end{array}$ & $\begin{array}{l}0.900 \\
0.013^{\star}\end{array}$ & & \\
\hline Ki67 & 11 & $\begin{array}{l}100.0 \pm \\
0.0\end{array}$ & & & \\
\hline$\leq 30 \%$ & $\begin{array}{l}23 \\
36\end{array}$ & $\begin{array}{l}88.2 \pm \\
5.5\end{array}$ & & & \\
\hline H ra & 43 & $90 \pm 9.5$ & & & \\
\hline $\begin{array}{l}\text { Hashimoto's } \\
\text { thyroiditis }\end{array}$ & 26 & $\begin{array}{l}87.5 \pm \\
5.2\end{array}$ & & & \\
\hline+ & 31 & $\begin{array}{l}87.2 \pm \\
5.4\end{array}$ & & & \\
\hline $\begin{array}{l}\text { Hypothyroidism } \\
\text { - }\end{array}$ & $\begin{array}{l}28 \\
41\end{array}$ & $\begin{array}{l}90.9 \pm \\
8.7^{-1}\end{array}$ & & & \\
\hline$*, P<0.05$, difference & s stati & $\begin{array}{l}95.5 \pm \\
\text { âl }\end{array}$ & & & \\
\hline
\end{tabular}




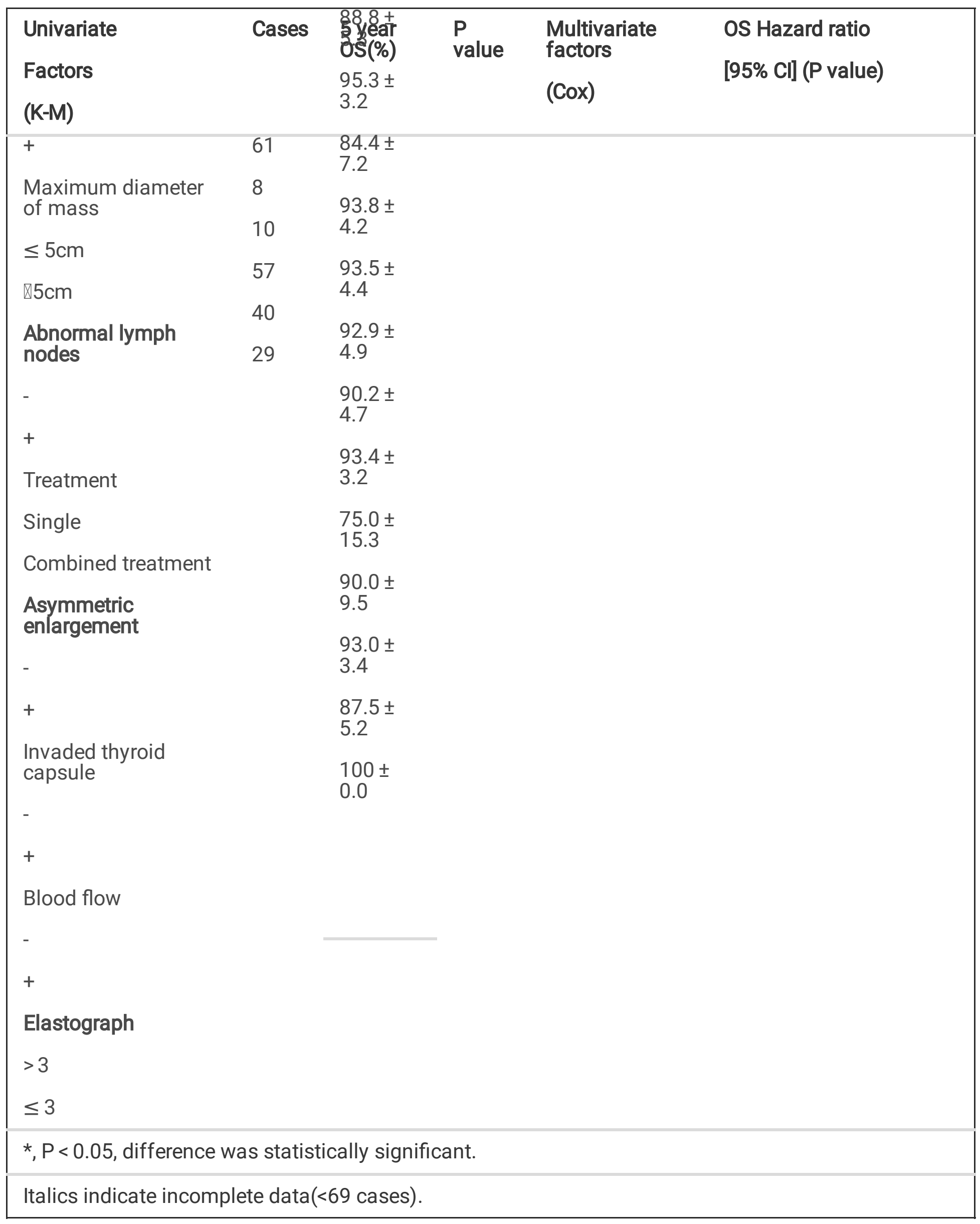




\section{Discussion}

Almost all PTLs are B-cell lymphomas which express the B-cell markers, CD20, CD79a, and Pax-5, whereas T-cell lymphomas are rare[14]. Our cases were all non-Hodgkin's lymphoma, and the proportion of female patients was relatively high, with an average age of $62 \pm 11$ years. The age of aggressive PTL was older when compared with indolent PTL, and most had elevated LDH levels which was consistent with previous reports $[1,15]$. Therefore, when examining older patients at initial consultation for Hashimoto's disease, clinicians must be aware of a high risk for PTL in these patients. DLBCL and MALT tumors were the most common PTL pathological types. In this study, the proportion of DLBCLs was the highest (51\%), followed by MALT (33\%). MALT-type lymphomas were mostly localized lesions, with low malignancy and a good prognosis, while DLBCLs were prone to metastasis and highly malignant. Burkitt lymphoma and follicular lymphoma are rare in PTL; their histological appearance and immunophenotypes are basically consistent with intra-lymph node lesion characteristics[12]. DLBCL combined with MALT indicated a transformed lymphoma, which was more aggressive than DLBCL alone, suggesting MALT lymphoma could be transformed into higher-grade, more aggressive lymphomas[16]. The mainstay treatment is combined modality therapy (CMT) for aggressive lymphoma and radiotherapy(RT) for localized indolent lymphoma. It was reported that CMT improved the prognosis of patients with aggressive lymphoma, while CMT for indolent lymphoma remains uncertain[3]. Multi-modal ultrasound follow-up may be helpful for early differentiated aggressive PTL from indolent PTL among patients with Hashimoto's disease.

PTL exhibits characteristic sonographic and doppler flow features which show central blood flow patterns highly suggestive of PTL rather than nodular goiter[17]. However, some sonographic features, such as asymmetric goiter with marked hypoechogenicity, internal cord-like or grid-like hyperechoic, enhanced posterior echo, large size, absent calcification and hypervascularity, and aspect ratio is not misaligned, etc., are different from most solid malignant lesions $[18,19]$. While PTL is rarely observed, my study had focused on multi-modal ultrasound. Cases in this study were divided into nodular, diffuse, and mixed types based on ultrasound image characteristics from previous reports[20]. The mixed type was more prone to aggressive lymphoma, and was mainly based on lesion distribution and morphology, suggesting different PTL types may be manifested as different stages of lymphoma growth and development[17, 21]. A previous study[22] reported that CEUS for PTL indicated a diffuse, non-uniform filling type, just like the snowflake enhancement observed in our study. Yang et al.[23] reported that CEUS was an efficient diagnostic tool for the differential diagnosis of PTL and nodular Hashimoto's thyroiditis for patients with diffuse HT. Previous reports have focused on nodular elastography for PTL. Wang et al. reported that along with several other parameters, a central blood flow pattern was highly suggestive of a PTL diagnosis rather than nodular goiter[17]. Nodular blood flow signals in aggressive PTL exhibited higher grades (usually $2-3$ ) than indolent PTL (generally $0-2$ ) in our study.

Previous studies showed that for thyroid lymphoma, core needle biopsy (CNB) sensitivity and accuracy were higher than fine-needle aspiration(FNA) for both preoperative and postoperative patients, and that CNB reduced unnecessary diagnostic surgery[24, 25]. In our study, four patients underwent FNA, 
suggesting that malignant tumors and lymphomas were to be examined, and then CNB was diagnosed, suggesting that conventional thyroid FNA may be limited in the diagnosis of PTL due to the limited number of cells obtained and the limitation of immunohistochemical technology. In addition, multi-modal ultrasound provided more information on PTL lesions for puncture, therefore CNB guided by multi-modal ultrasound is more advantageous for diagnosing PTL when compared with FNA or surgery.

PTL combined with thyroid cancer is very rare[26, 27]. In this study, we observed five PTL cases with PTC, maximum age 62, and four cases are MALT lymphoma with HT and diffuse type of ultrasound pattern. Interestingly, the prognosis was not worsened by combined PTC and PTL, but was more likely affected by the more poorly staged tumor. Thus, for patients with concomitant PTC and PTL, treatments were conducted which prioritized the tumor with the worse prognosis and/or the worse stage at diagnosis[28]. Graceffa et al. [29] expounded the frequent coexistence of PTC and HT. Similarly, the probability of PTL in HT populations was approximately 50 times higher than the general population[1, 9]. This situation highlights the importance of monitoring HT patients, particularly if they have long-lasting disease with nodules. Patients with coexisting PTC and PTL require a multidisciplinary approach for diagnostic workup and optimal management.

PTL has a good prognosis, which is closely related to the pathological type and clinical stage, especially tumor size and extent of cancer invasion. MALT is an indolent lymphoma with a 5-year survival rate of $62-96 \%$, while DLBCL is an aggressive lymphoma with a 5 -year survival rate of $45-75 \%[30]$. Cha et al. [31]reported that the 5-year OS and PFS rate of aggressive PTL was $75.6 \%$ and $73.9 \%$, respectively. Watanabe et al. [32]found that five-year overall survival and event-free survival for elderly patients with thyroid DLBCL were $87 \%$ (95\% confidence interval [95\% Cl], 64-96\%) and 74\% (95\% Cl, 50-89\%), respectively. In this study, the OS of indolent PTL patients was significantly better than aggressive PTL patients $(P<0.05)$. PTL patients with age $>70$ years old, aggressive pathology, Ki67 $>30 \%$, elastography scored $>3$ are more likely to have poor prognosis. Multivariable cox regression analysis showed that age was an independent risk factor for PTL. Watanabe et al. [33] in another research reported that higher age and higher erythrocyte sedimentation rate (ESR) were significant risk factors for OS, which is almost similar to our research.

Our study had limitations. Firstly, PTL is rare and the single-center nature of our study meant our cohort was small. Secondly, to precisely identify histopathological PTL types over a long period, only patients with a pathologically proven diagnosis were included in this study. Also, some cases were lost to followup. However, PTL is relatively rare, therefore, it is difficult to perform prospective studies and identify large numbers for analysis.

In summary, the application of multiple ultrasound examination modes is beneficial for capturing disease information and PTL ultrasound patterns. Also, the approach comprehensively evaluates tumor boundaries, sizes, shapes, internal echo, hemodynamics, spatial structures, soft textures, and pathological tissues. In addition, PTL patients with age > 70 years old, aggressive pathology, Ki67 > 30\%, elastography scored $>3$ are more likely to have poor prognosis. It is recommended elderly patients with 
HT are closely followed-up by multi-mode ultrasound to improve PTL early detection and strategically target aggressive tumors.

\section{Abbreviations}

PTL: primary thyroid lymphoma; DLBCL: diffuse large B cell lymphoma; MALT: mucosa-associated lymphoid tissue lymphoma ; FLIII: follicular lymphoma III; CEUS: Contrast-enhanced ultrasonography; HT: Hashimoto's thyroiditis;

CNB: core needle biopsy ; FNA: fine-needle aspiration; CMT: combined modality therapy ; OS: overall survival; PFS: progression-free survival; HR: Hazard Ratio; Cl: Confidence Interval

\section{Declarations}

\section{Funding}

This study is funded by the National Natural Science Foundation of China(No.81871370, No.82071946) and Zhejiang Provincial Natural Science Foundation of China(No.LSD19H180001)

\section{Author contributions}

CJ Peng and C Yang: protocol development, data collection, data analysis, and manuscript writing; JC Yao and LY Zhou: protocol development and ultrasonic data collection; JZ Wu: statistical analysis; JJ Xu: pathology reading and selection: JZ Zhao and SL Peng: protocol development and clinical information collection; D Xu: protocol development, manuscript writing/editing.

Conflict of interest: The authors declare that they have no conflict of interest.

\section{Ethics approval and consent to participate}

This study was approved by the Ethics Committee of Zhejiang Cancer Hospital. The need for informed consent from all patients was waived due to the study's retrospective nature.

\section{Availability of data and materials}

Our data can not be made publicly available for ethical reasons. Data are from the present study whose authors may be contacted at pengcj@zjcc.org.cn or Department of Ultrasound Medicine, Zhejiang Cancer Hospital, Hangzhou, China

\section{References}

1. Travaglino, A., et al., Hashimoto Thyroiditis in Primary Thyroid Non-Hodgkin Lymphoma. Am J Clin Pathol, 2020. 153(2): p. 156-164. 
2. Stein, S.A. and L. Wartofsky, Primary thyroid lymphoma: a clinical review. J Clin Endocrinol Metab, 2013. 98(8): p. 3131-8.

3. Pavlidis, E.T. and T.E. Pavlidis, A Review of Primary Thyroid Lymphoma: Molecular Factors, Diagnosis and Management. J Invest Surg, 2019. 32(2): p. 137-142.

4. Z, L., et al., Differences in Multi-Modal Ultrasound Imaging between Triple Negative and Non-Triple. 2016. p. 882-890.

5. Z, Y., et al., - Preoperative diagnosis and prediction of hepatocellular carcinoma: Radiomics. - BMC Cancer. 2018 Nov 12;18(1):1089. doi: 10.1186/s12885-018-5003-4., (- 1471-2407 (Electronic)): p. 1089.

6. T, D., et al., - lanalumab (VAY736) in primary Sjögren's syndrome: assessing disease activity using. Clin Exp Rheumatol. 2020 Jul-Aug;38 Suppl 126(4):228-236. Epub 2020 Oct 23., (- 0392-856X (Print)): p. $-228-236$.

7. SH, S., et al., - The 2016 revision of the World Health Organization classification of lymphoid. - Blood. 2016 May 19;127(20):2375-90. doi:

10.1182

/blood-2016-01-643569. Epub 2016 Mar, (- 1528-0020 (Electronic)): p. - 2375-90.

8. RMS, S., et al., - Ultrasound Elastography: Review of Techniques and Clinical Applications. Theranostics. 2017 Mar 7;7(5):1303-1329. doi: 10.7150/thno.18650. eCollection 2017., (- 18387640 (Electronic)): p. - 1303-1329.

9. Xi, X., et al., Differentiation of Thyroid Nodules Difficult to Diagnose With Contrast-Enhanced Ultrasonography and Real-Time Elastography. Front Oncol, 2020. 10: p. 112.

10. Jain, N.K., et al., Extrathyroidal Extension is an Important Predictor of Regional Lymph Node Metastasis in Pediatric Differentiated Thyroid Cancer. Thyroid, 2020. 30(7): p. 1037-1043.

11. Sidhu, P.S., et al., The EFSUMB Guidelines and Recommendations for the Clinical Practice of Contrast-Enhanced UItrasound (CEUS) in Non-Hepatic Applications: Update 2017 (Long Version). Ultraschall Med, 2018. 39(2): p. e2-e44.

12. Hayashi, T., et al., Clinicopathological features of primary thyroid Burkitt's lymphoma: a systematic review and meta-analysis. Diagn Pathol, 2020. 15(1): p. 13.

13. $\mathrm{H}, \mathrm{O}$., et al., Usefulness of ultrasonography for diagnosis of malignant lymphoma of the thyroid. Thyroid, 2006. 16(10): p. 983-7.

14. Czopnik, P., et al., Primary thyroid lymphoma: a rare but challenging diagnosis. Pol Arch Intern Med, 2017. 127(5): p. 361-364.

15. Chai, Y.J., et al., Clinicopathological characteristics and treatment outcomes of 38 cases of primary thyroid lymphoma: a multicenter study. Ann Surg Treat Res, 2015. 89(6): p. 295-9.

16. Derringer, G.A., et al., Malignant lymphoma of the thyroid gland: a clinicopathologic study of 108 cases. Am J Surg Pathol, 2000. 24(5): p. 623-39. 
17. Wang, Z., et al., Primary thyroid lymphoma has different sonographic and color Doppler features compared to nodular goiter. J Ultrasound Med, 2015. 34(2): p. 317-23.

18. Gu, L.S., et al., Comparison of sonographic characteristics of primary thyroid lymphoma and anaplastic thyroid carcinoma. J Thorac Dis, 2017. 9(11): p. 4774-4784.

19. Li, P. and H. Zhang, Ultrasonography in the Diagnosis and Monitoring of Therapy for Primary Thyroid Lymphoma. Ultrasound Q, 2019. 35(3): p. 246-252.

20. Mizokami, T., et al., Development of Primary Thyroid Lymphoma during an Ultrasonographic Followup of Hashimoto's Thyroiditis: A Report of 9 Cases. Intern Med, 2016. 55(8): p. 943-8.

21. Yang, L., et al., 12 cases of primary thyroid lymphoma in China. J Endocrinol Invest, 2015. 38(7): p. $739-44$.

22. Wei, X., et al., Evaluation of primary thyroid lymphoma by ultrasonography combined with contrastenhanced ultrasonography: A pilot study. Indian J Cancer, 2015. 52(4): p. 546-50.

23. Yang, L., et al., Contrast-Enhanced Ultrasound in the Differential Diagnosis of Primary Thyroid Lymphoma and Nodular Hashimoto's Thyroiditis in a Background of Heterogeneous Parenchyma. Front Oncol, 2020. 10: p. 597975.

24. Wolinski, K., A. Stangierski, and M. Ruchala, Comparison of diagnostic yield of core-needle and fineneedle aspiration biopsies of thyroid lesions: Systematic review and meta-analysis. Eur Radiol, 2017. 27(1): p. 431-436.

25. Chatani, S., et al., Image-guided core needle biopsy in the diagnosis of malignant lymphoma: comparison with surgical excision biopsy. Eur J Radiol, 2020. 127: p. 108990.

26. Shen, G., et al., Coexistence of Papillary Thyroid Carcinoma With Thyroid MALT Lymphoma in a Patient With Hashimoto's Thyroiditis: A Clinical Case Report. Medicine (Baltimore), 2015. 94(52): p. e2403.

27. Vassilatou, E., et al., Coexistence of differentiated thyroid carcinoma with primary thyroid lymphoma in a background of Hashimoto's thyroiditis. J Clin Oncol, 2011. 29(25): p. e709-12.

28. Trovato, M., et al., Coexistence of diffuse large B-cell lymphoma and papillary thyroid carcinoma in a patient affected by Hashimoto's thyroiditis. Arch Endocrinol Metab, 2017. 61(6): p. 643-646.

29. Graceffa, G., et al., Association between Hashimoto's thyroiditis and papillary thyroid carcinoma: a retrospective analysis of 305 patients. BMC Endocr Disord, 2019. 19(Suppl 1): p. 26.

30. Walsh, S., et al., Thyroid lymphoma: recent advances in diagnosis and optimal management strategies. Oncologist, 2013. 18(9): p. 994-1003.

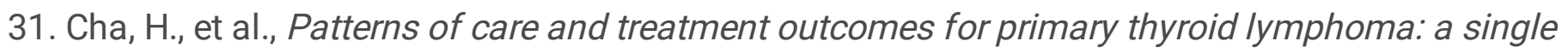
institution study. Radiat Oncol J, 2013. 31(4): p. 177-84.

32. Watanabe, N., et al., Rituximab-including combined modality treatment for primary thyroid lymphoma: an effective regimen for elderly patients. Thyroid, 2014. 24(6): p. 994-9.

33. Watanabe, N., et al., Clinicopathological features of 171 cases of primary thyroid lymphoma: a longterm study involving 24553 patients with Hashimoto's disease. Br J Haematol, 2011. 153(2): p. 236- 


\section{Figures}

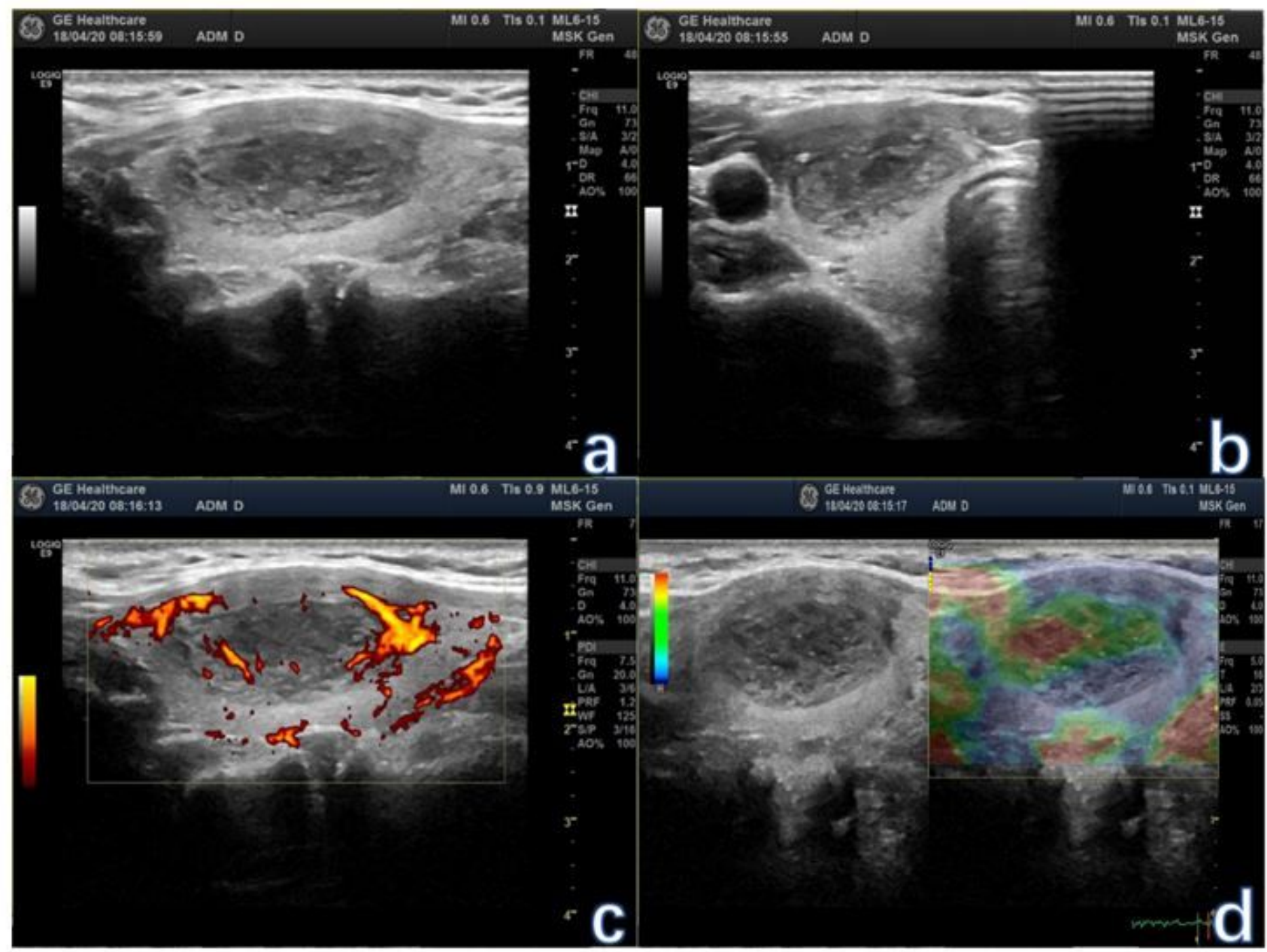

\section{Figure 1}

Multi-modal ultrasonic images of PTL (Burkitt, nodular ultrasonic pattern). a. longitudinal section twodimensional (2D) ultrasonography, b. transverse section 2D ultrasonography, c. longitudinal section energy Doppler flow imaging, d. ultrasonic elastography, lesion elastography color coded image show three point (soft). 


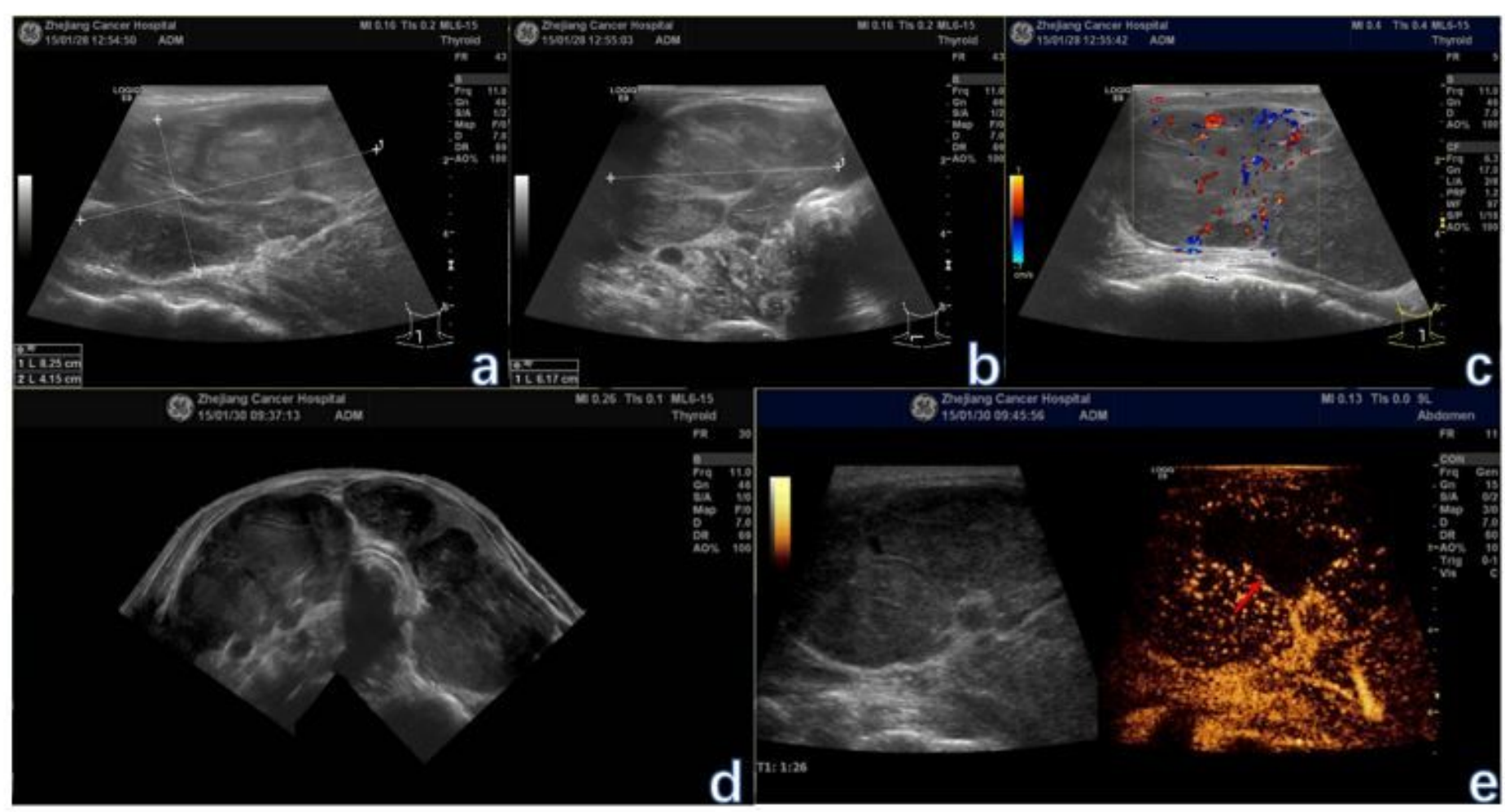

\section{Figure 2}

Multi-modal ultrasound of PTL (DLBCL, mixed ultrasonic pattern). a. longitudinal two-dimensional (2D) ultrasound image, b. transverse 2D ultrasound image, c. color Doppler flow imaging, d. wide-range imaging ultrasound image, e. contrast-enhanced ultrasound performance, lesions show diffuse nonuniform mild enhancement (snowflake enhancement), local visible a little echo-free zone (red arrow). 


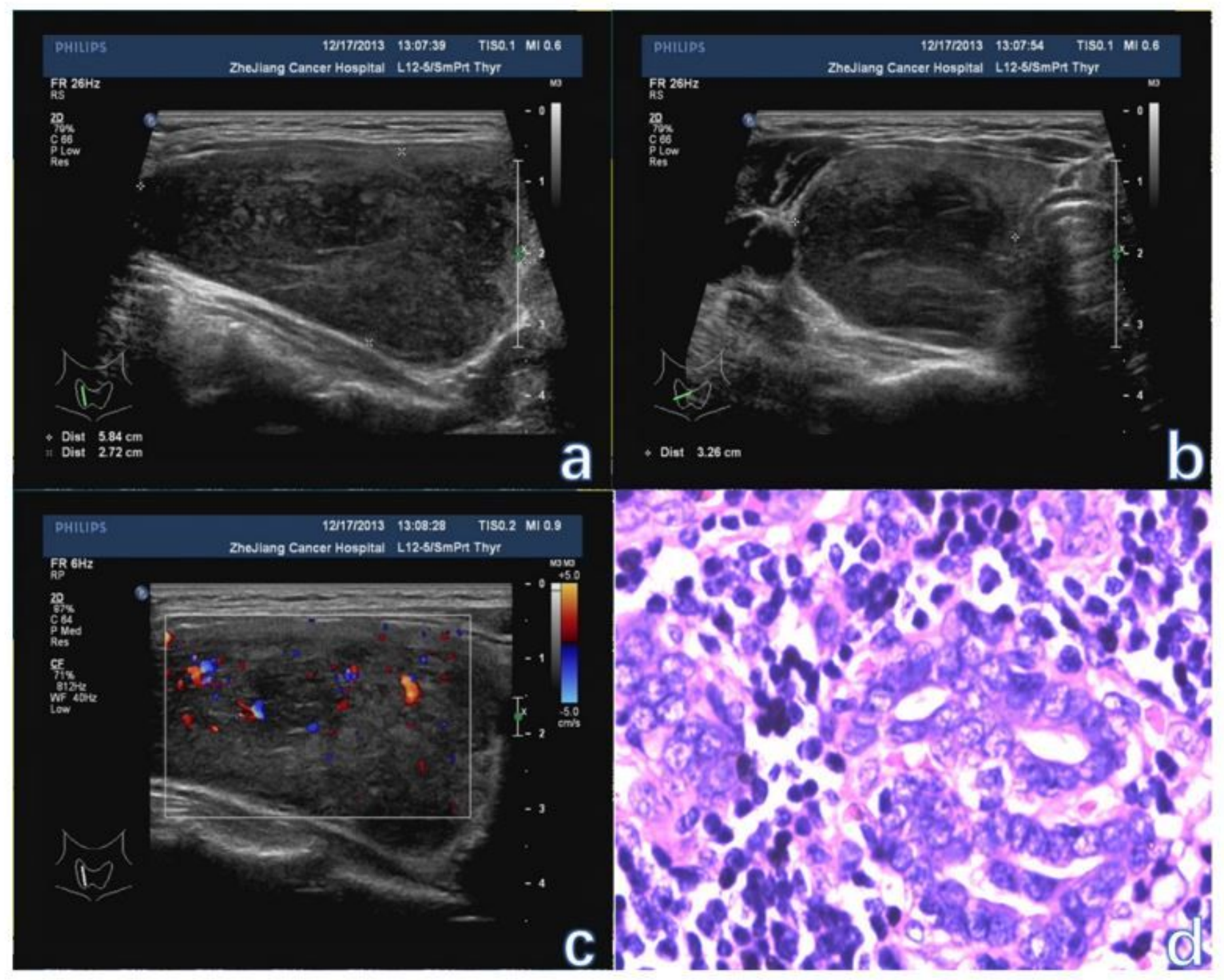

\section{Figure 3}

PTL with papillary carcinoma (MALT, diffuse ultrasonic pattern). a. longitudinal two-dimensional (2D) ultrasound, b. transverse 2D ultrasound, c. longitudinal color Doppler ultrasound image, d. pathological section $(\times 400)$. 

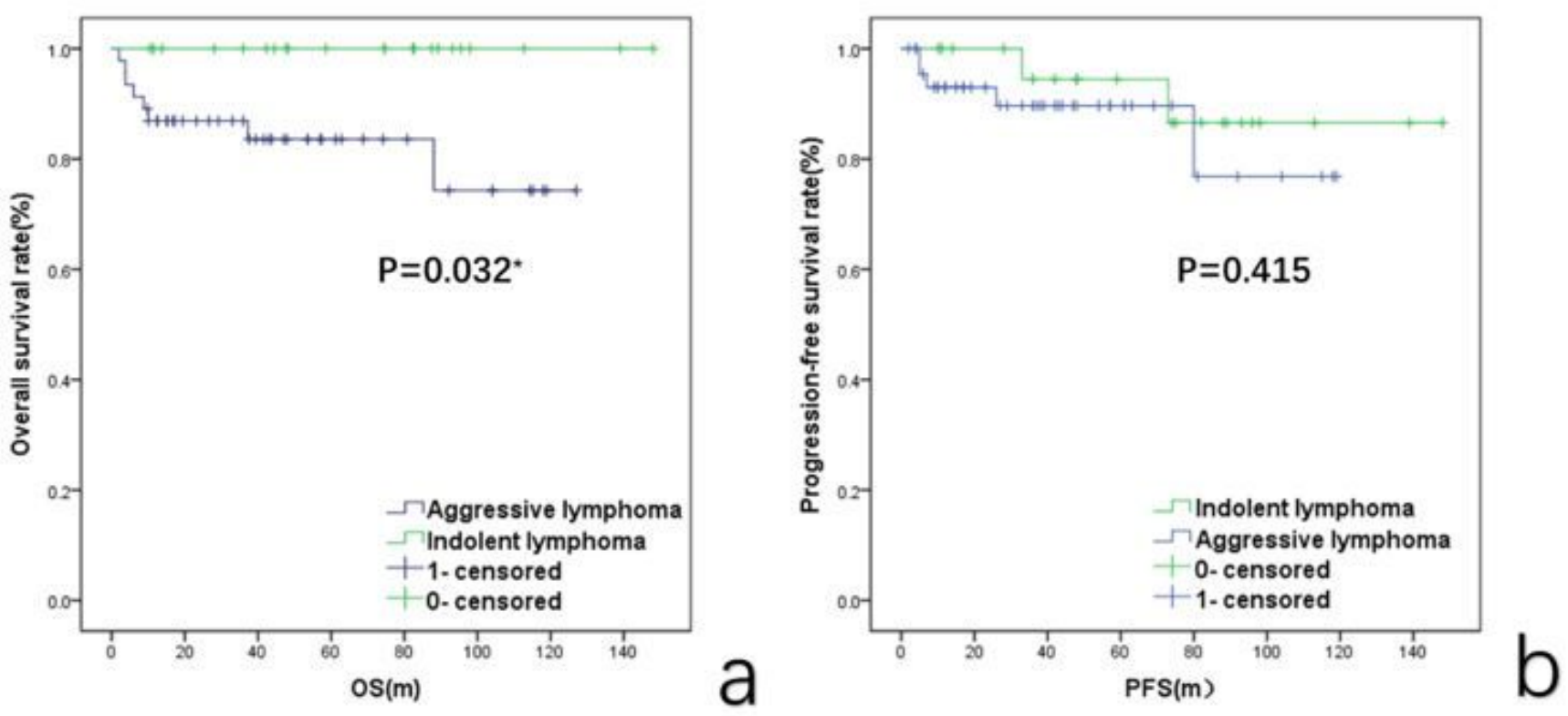

Figure 4

Survival curves for invasive and indolent primary thyroid lymphoma. a. Overall survival curve b. Progression-free survival curve 